The present state of the Analytical Eugine is as follows:

All the great principles on which the discovery rests have been explained, and drawings of mechanical structures have been made, by which each may be carried into operation.

Simpler mecisanisms, as well as more extensice principles than were required for the Difference Engine, have been discovered for all the elementary portions of the-Analytical Engine, and numerous drawings of these successive simplifications exist.

The mode of combining the various sections of which the Engine is formed has been examined with unceasing anxiety, for the purpose of reducing the whole combination to the greatest possible simplicity. Drawings of almost all the plans thus discussed have been made, and the latest of the drawings (bearing the number 28) shows how many have been superseded, and also, from its extreme comparative simplicity, that little further advance can be expected in that direction.
Mechanical notations have been made both of the actions of detached parts and of the general action of the whole, which cover about four or five hundred large folio sheets of paper.

The original rough sketches are contained in about five volumes.

There are upwards of one hundred large drawings.

No part of the construction of the Analytical Engine has yet been commenced. A long series of experiments have, however, been made upon the art of shaping metals; and the tools to be employed for that purpose have been discussed, and many drawings of them prepared. The great object of these inquirics and experiments is, on the one hand, by simplifying as much as possible the construction, and on the other, by contriving new and cheaper means of execution, at length to reduce the expense within those limits which a private individual may command.

\title{
Ueber die Anwendung der Methode der kleinsten Quadrate auf einen besondern Fall.
}

Von Hern Professor Argelander, Director der Sternwarte in Bonn.

Wenn aus einer oder mehreren Beobachtungsreihen eine sehr grofse Menge von unbekanuten Grörsen abzuleiten ist, so wird bekanntlich die directe Anwendung der Methode der kleinsten Quadrate wegen der übermäfsigen Ausdehnung der Rechnung selrr lïstig und oft ganz unmöglich, so dafs man in diesem Falle zur Ableitung von partiellen Resultaten für einzelne Unbekannte und successive Annäherung seine Zuflucht nehnnen mufs. In einzelnen Fällen indessen, in denen die $\mathbf{A b}$ hängigkeit der Beobachtungen von den unbekannten Gröfsen durch sehr symmetrische Formeln gegeben wird, läfst sich eine sehr bedeutende Abkürzung der Rechnung finden. $\mathbf{Z u}$ diesen gehört der bekannte und so häutig vorkommende, den Bessel in der Einleitung zum ersten Bande der Königsherger Beobachtungen behandelt hat; kürzlich ist mir ein auderer der Art vorgekommen, nämlich derjenige, wo die Unbekannten so von einander abhängen, dafs die Beobachtung uninittelbar die Summe oder Differenz jo zweier von ihnen giebt, und die Beobachtungen vollständig für alle verschiedenen Combinationen derselben zu je zweien vorhanden sind. Es seien also die einfachen aus den Beobachtungen entnommenen Gleichungen für die $(\mu+1)$ Unbekannten $p, q, r, s, t, \ldots y, z$

$$
\begin{array}{ccc}
p \pm q=\alpha^{\prime} & q \pm r=\beta^{\prime \prime} & r \pm s=\gamma^{\prime \prime \prime} \\
p \pm r=\beta^{\prime} & q \pm s=\gamma^{\prime \prime} & r \pm t=d^{\prime \prime \prime} \\
p \pm s=\gamma^{\prime} & q \pm t=d^{\prime \prime} & \vdots \\
p \pm t=\delta^{\prime} & \vdots & r \pm z=\lambda^{\prime \prime \prime} \\
p \pm z=\lambda^{\prime} & q \pm z=\lambda^{\prime \prime} & \left.y \pm z=\lambda^{\prime \prime}\right)
\end{array}
$$

in denen allen entweder das obere oder das untere Zeichen gilt, aber in allen dasselbe. Durch die Bedingung des Minimums für die Summe der Quadrate der Fehler entstehen hieraus die zur Auflüsung nüthigen $(\mu+1)$ Finalgleichungen

$$
\begin{gathered}
0=A+\mu p \pm q \pm r \pm \text { etc...... } \\
0=B \pm p+\mu q \pm r \pm \text { etc..... } \\
\vdots \\
0=K \pm p \pm q \pm r \pm \text { etc..... } \\
0=x \pm \mu y \pm z
\end{gathered}
$$

Es sind nun die beiden Fälle zu unterscheiden:

I. Wenn überall das obere Zeichen gilt, so ist

$$
\begin{gathered}
A=-\left[\alpha^{\prime}+\beta^{\prime}+\gamma^{\prime}+\cdots+\lambda^{\prime}\right] \\
B=-\left[\beta^{\prime}+\beta^{\prime \prime}+\gamma^{\prime \prime}+\cdots+\lambda^{\prime \prime}\right] \\
\quad \text { etc. etc. } \\
I=-\left[\lambda^{\prime}+\lambda^{\prime \prime}+\lambda^{\prime \prime \prime}+\cdots+\lambda^{\prime \prime \prime}\right]
\end{gathered}
$$

Nennt man nun die Summe sämmtlicher Unbekannten, also $(p+q+r+\cdots+z)=S$, so verwandeln sich die obigen Gleichungen in die folgenden

$$
\begin{aligned}
0=A+(\mu-1) p+S \\
0=B+(\mu-1) q+S \\
\quad \text { etc. etc. } \\
0=L+(\mu-1) 2+S
\end{aligned}
$$

addirt man diese $(\mu+1)$ Gleichungen zusammen, so erhält man

$$
0=(A+B+\cdots+L)+(\mu-1) S+(\mu+1) S
$$


hieraus ferner

$$
S=-\frac{(A+B+\cdots+L)}{2 \mu}
$$

und endlich, da offenbar $S$ bekannt ist

$$
p=-\frac{A+S}{\mu-1} ; \quad q=-\frac{B+S}{\mu-1} ; \text { etc....z }=-\frac{L+S}{\mu-1},
$$

so dafs also die ganze Rechnung sich anf einfache Additionen und die Division durch $(\mu-1)$ reducirt. Man gelangt zu denselben Formeln auch durch das gewöholiche Eliminationsverfahren, wodurch aber nebenbei noch die Werthzahlen bestimmt werden. Wenn wir nämlich die bekannten Gaussischen Bezeichnungen für die Summen der Quadrational-Producte der Coëfficienten, so wie für ihre Umformungen durch die successiven Eliminationen beibehalten, ferner annehmen, dafs die Elimination mit $z$ heginne, und bei der vten diejenige Unbekannte climinirt werde, deren Coëfficienten bei der gewöhnlichen Auflösung mit $f$ bezeichnet werden, so dafs also $\left(f_{n}\right)=T$ ist; so sind offenbar alle $(a b),(a c), \ldots(b c)$ etc.... $=1$, alle $(a a),(b b),(c c)$ etc. $=\mu$, und folglich auch $\left(a b_{\nu}\right)=\left(a c_{\nu}\right)=$ etc. $\ldots=\left(b c_{v}\right)=$ etc.... und eben so auch alle $\left(a a_{\nu}\right)=\left(b b_{\nu}\right)$ $\left(c c_{v}\right)=$ etc.... Es wird danu, wie man ohue Mühe findet,

$$
\begin{aligned}
& A_{v}=\left(a n_{\nu}\right)=A-(F+G+\text { etc.... }+L):(\mu+\nu-1) \\
& B_{\nu}=\left(b n_{\nu}\right)=B-(F+G+\text { etc.... }+2):(\mu+\nu-1) \\
& \text { etc. etc. etc. } \\
& \left(a b_{\nu}\right)=\left(a c_{\nu}\right)=\text { etc.... }=(\mu-1):(\mu+\nu-1) \\
& \left(a a_{\nu}\right)=\left(b b_{\nu}\right)=\text { etc. } \ldots=(\mu-1) \cdot(\mu+\nu):(\mu+\nu-1)
\end{aligned}
$$

Setzt man nun $\nu=\mu$, so erhält man als Endresultat

$A \mu=A-(B+c+$ etc....

$B \mu=B-(A+c+$ etc. . . + $L) \cdot(2 \mu-1)=2 \mu(B+S):(2 \mu-1)$

$$
\text { etc. etc. }
$$

die Werthzahlen

$\left(a a_{\mu}\right)=\left(b b_{\mu}\right)=$ etc. $\ldots=(\mu-1) 2 \mu:(2 \mu-1)$

und daraus für die Unbekannten genau die früher gefundenen Werthe, da bekanntlich $p=-A_{\mu}:\left(a a_{\mu}\right) ; q=-B_{\mu}:\left(b b_{\mu}\right)$ etc. ist.

II. Im zweiten Falle wird die Aufgabe bekanntlich unbestimmt, und lifst sich nur auflösen, wenn man für eine der Unbekannten einen willkürlichen Werth annimmt, oder die andern als Functionen dieser entwickelt. Es möge dies $\approx$ scin; so fällt die letzte der Finalgleichungen fort, und die übrigbleibenden, $\mu$ an der Zahl, verwandeln sich, weun man $A-z=A^{\prime}, \quad B-z=B^{\prime}, \ldots K-z=K^{\prime}$ setzt, in die folgenden

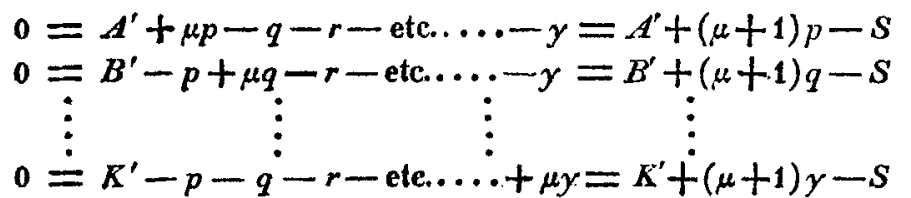

wo $S$ jetzt die Summe der $\mu$ Unbekannten $p+q+\ldots y$ ist. Durch Addition sämmtlicher $\mu$ Gleichungen erhält man ferner

$$
0=A^{\prime}+B^{\prime}+\ldots+K^{\prime \prime}+(\mu+1) S-\mu S
$$

also

$$
S=-\left[A^{\prime}+B^{\prime}+\text { etc.... } K^{\prime}\right]=-[A+B+\ldots+K]+\mu z
$$
und

$$
\begin{gathered}
p=\left(S-A^{\prime}\right):(\mu+1)=-[A+(A+B+\ldots+K)]:(\mu+1)+z \\
q=\left(S-B^{\prime}\right):(\mu+1)=-[B+(A+B+\ldots+K)]:(\mu+1)+z \\
\text { etc. } \quad \text { etc. etc. }
\end{gathered}
$$

setzt man also die Summe $(A+B+\ldots+K)=S^{\prime}$, so erhält man

$$
\begin{gathered}
p-z=-\left(A+S^{\prime}\right):(\mu+1) \\
q-z=-\left(B+S^{\prime}\right):(\mu+1) \\
\text { etc. }
\end{gathered}
$$

Man erhält also hier die Unterschiede aller übrigen $\mathbf{U}_{n-}$ bekannten von $Z$, und daher, wenn man tür dieses einen bestimmten Werth setzt, jene selbst. Es wird am bequemsten seyn, $z=0$ zu setzen, wodurch die Rechnung am einfach. sten wird. Will man für $A, B$, etc... die kleinsten Zahlen haben, so wähle man fir $z$ diejenige Unbekannte, die in der Mitte zwischen den anderu der Gröfse nach liegt; wählt man dafür die kleinste, so erhält man für die andern lauter positive Werthe, Die Quantititen $A, B$, etc. erhalten hier übrigens folgende Werthe:

$$
\begin{aligned}
& A=-\left(\alpha^{\prime}+\beta^{\prime}+\gamma^{\prime}+\ldots .+\lambda^{\prime}\right) \\
& B=-\left(-\alpha^{\prime}+\beta^{\prime \prime}+\gamma^{\prime \prime}+\ldots .+\lambda^{\prime \prime}\right) \\
& C=-\left(-\beta^{\prime}-\beta^{\prime \prime}+\gamma^{\prime \prime}+\ldots .+\lambda^{\prime \prime}\right) \\
& \quad \text { etc. } \quad \text { etc. } \\
& K=-\left(-x^{\prime}-x^{\prime \prime} \text { etc.... }-x^{(\mu-1)}+\lambda^{(\mu)}\right)
\end{aligned}
$$

Dicselben Werthe für die Unbekannten giebt auch die andere Ableitung; sie giebt aber aufserdem noch die Werthzahlen, welche hier, da eine Unbekannte fortfällt, bei der $(\mu-1)^{\text {ten }}$ Elimination erfolgen, und natürlich nur für die relative Bestimmung gegen z gelten. Man erhält

$$
\left(a a_{\mu-1}\right)=\left(b b_{u-1}\right)=\text { etc. } \ldots=\frac{1}{2}(\mu+1) \text {. }
$$

Diese zweite Form der Aufgabe erhält ihre Anwendung unter andern, wenu man eiıe Reihe von Sternen, jeden mit allen andern, in Bezichung auf ihre Helligkeit verglichen hat, und daraus ihre relative Helligkeit gegen einen derselben sucht. Sind nun hier, wie es wohl meistens zutreffen wird, nicht alle Sterne mit alleı verglichen, so werden die Finalgleichungen unvollständig; es werden nicht in allen alle Unbekaunte vor. kommen, und $(a a),(b b)$ etc. werden nicht alle $=\mu$ werden, sondern einige oder auch alle kleiner. In diesem Falle lärst sich also die gegebene Auflösung direct nicht anwenden. Wenn aber der ausgefallenen Gleichungen nicht gar zu viele sind, so wird man durch Annäherungen sehr rasch zum Ziele gelangen. 
Man nehme vorläufige Werthe für die Unbekanten an, wie sie die einzelnen Gleichungen ergeben, wenn man $z=0$ setzt, also z. B. flïr $\rho$ den Werth $\left(\lambda^{\prime}+\lambda^{\prime \prime}+\alpha^{\prime}+\lambda^{\prime \prime \prime}+\beta^{\prime}+\right.$ etc. $): \mu$, oder noch besser $\left(2 \lambda^{\prime}+\lambda^{\prime \prime}+\alpha^{\prime}+\lambda^{\prime \prime}+\beta^{\prime}+\right.$ etc....) $:(\mu+1)$, in. den , dieser geradezu der wahrscheinlichste Werth ist, wenn alle Verbindungen zwischen $p$ und den andern Unbekannten beobachtet sind. Unter Annahme dieser Werthe vervollständige man die Finalgleichungen, indem man für jede fehlende Unbekannte die Quantitäten $A, B$, etc., um deren angenommene numerische Werthe vermindert, daraus nach den gegebenen Vorschriften neue Werthe für die Unbekannten bestimmt, durch diese neuen Werthe die $A, B$, etc.... wieder corrigirt, und so fortfährt, bis die in den uncorrigirten Finalgleichungen übrigbleihenden Fehler so klein werden, dals man sie für den vorgesetzten $Z$ weck als verschwindend betrachten kann. Wie man leicht übersieht, sind die Unterschiede der auf diese Weise erhaltenen successiven Werthe von $A, B$, etc. den $A$ brweichungen der Finalgleichungen in den verschiedenen Hypothesen von 0 , also den Fehlern, gleich, und man kann daher ohne weitere Rechnung aus diesen Unterschieden dic Gröfse der Annäherung an die Wahrheit, die man erreicht hat, beurtheilen.
Argelander.

Scheinbare Positionen der bei den Beobachtungen des Mauvaisschen Cometen auf der Hamburger Sternwartc gebrauchten Vergleichungs-Sterne.

Von Ilerm Director Rimcker mit dem Repsoldschen Meridiankreise bestimmt.

\begin{tabular}{|c|c|c|}
\hline $\begin{array}{l}\text { Mag der Ver- } \\
\text { Mleichung. } \\
1843 .\end{array}$ & $\begin{array}{l}\begin{array}{c}\text { Scheinb. AIR. } \\
\text { in Zeit. } \\
\underbrace{}_{22^{\mathrm{h}} 34^{\prime}} 25^{\prime \prime} 420\end{array}\end{array}$ & $\underbrace{\text { Scheinb. Decl. }}_{28^{\circ} 29^{\prime} 21^{\prime \prime} 55}$ \\
\hline Mai 29 & $\begin{array}{rrr}22 & 39 & 30,522 \\
22 & 39 & 59,492 \\
22 & 41 & 5,387\end{array}$ & $\begin{array}{lll}27 & 41 & 38,92 \\
27 & 25 & 59,36 \\
27 & 17 & 44,16\end{array}$ \\
\hline Mai 30 & $\begin{array}{rrr}22 & 44 & 3,042 \\
22 & 46 & 44,431 \\
22 & 47 & 27,785\end{array}$ & $\begin{array}{rrr}27 & 10 & 10,47 \\
27 & 11 & 3,24 \\
27 & 10 & 28,54\end{array}$ \\
\hline Juni 4 & $\begin{array}{lll}22 & 51 & 24,376 \\
22 & 51 & 28,271 \\
22 & 52 & 44,974\end{array}$ & $\begin{array}{lll}26 & 39 & 39,73 \\
26 & 54 & 26,12 \\
26 & 44 & 29,68\end{array}$ \\
\hline Juni 6 & $\begin{array}{rrr}22 & 52 & 57,708 \\
22 & 53 & 39,193 \\
22 & 57 & 46,813 \\
23 & 0 & 13,769 \\
23 & 1 & 34,172 \\
23 & 4 & 14,535\end{array}$ & $\begin{array}{rrr}26 & 13 & 39,80 \\
26 & 9 & 19,87 \\
26 & 8 & 15,29 \\
26 & 6 & 55,03 \\
26 & 3 & 51,49 \\
26 & 0 & 5,00\end{array}$ \\
\hline Juni 18 & $\begin{array}{rrr}23 & 8 & 0,729 \\
23 & 8 & 16,538\end{array}$ & $\begin{array}{rrr}23 & 34 & 14,96 \\
23 & 55 & 2,66\end{array}$ \\
\hline Juni 19 & $\begin{array}{lll}23 & 14 & 21,284 \\
23 & 14 & 46,304 \\
23 & 19 & 13,900\end{array}$ & $\begin{array}{lll}23 & 25 & 39,10 \\
23 & 14 & 35,51 \\
23 & 36 & 20,95\end{array}$ \\
\hline Juui 20 & $\begin{array}{llll}23 & 1 & 58,612 \\
23 & 12 & 55,040 \\
23 & 14 & 46,337 \\
23 & 16 & 35,548 \\
23 & 16 & 35,681\end{array}$ & $\begin{array}{rrr}22 & 58 & 15,95 \\
22 & 53 & 2,58 \\
23 & 14 & 35,73 \\
23 & 8 & 7,23 \\
22 & 54 & 3,90\end{array}$ \\
\hline Juni 23 & $\begin{array}{lll}23 & 17 & 35,943 \\
23 & 21 & 19,476\end{array}$ & $\begin{array}{lll}22 & 32 & 33,39 \\
22 & 11 & 23,37\end{array}$ \\
\hline Juni 26 & $\begin{array}{lll}23 & 21 & 29,082 \\
23 & 22 & 13,075 \\
23 & 24 & 57,577 \\
23 & 25 & 39,549 \\
23 & 25 & 47,686\end{array}$ & $\begin{array}{rrr}21 & 30 & 15,92 \\
21 & 21 & 1,17 \\
21 & 25 & 49,29 \\
21 & 38 & 7,37 \\
21 & 27 & 53,69\end{array}$ \\
\hline
\end{tabular}

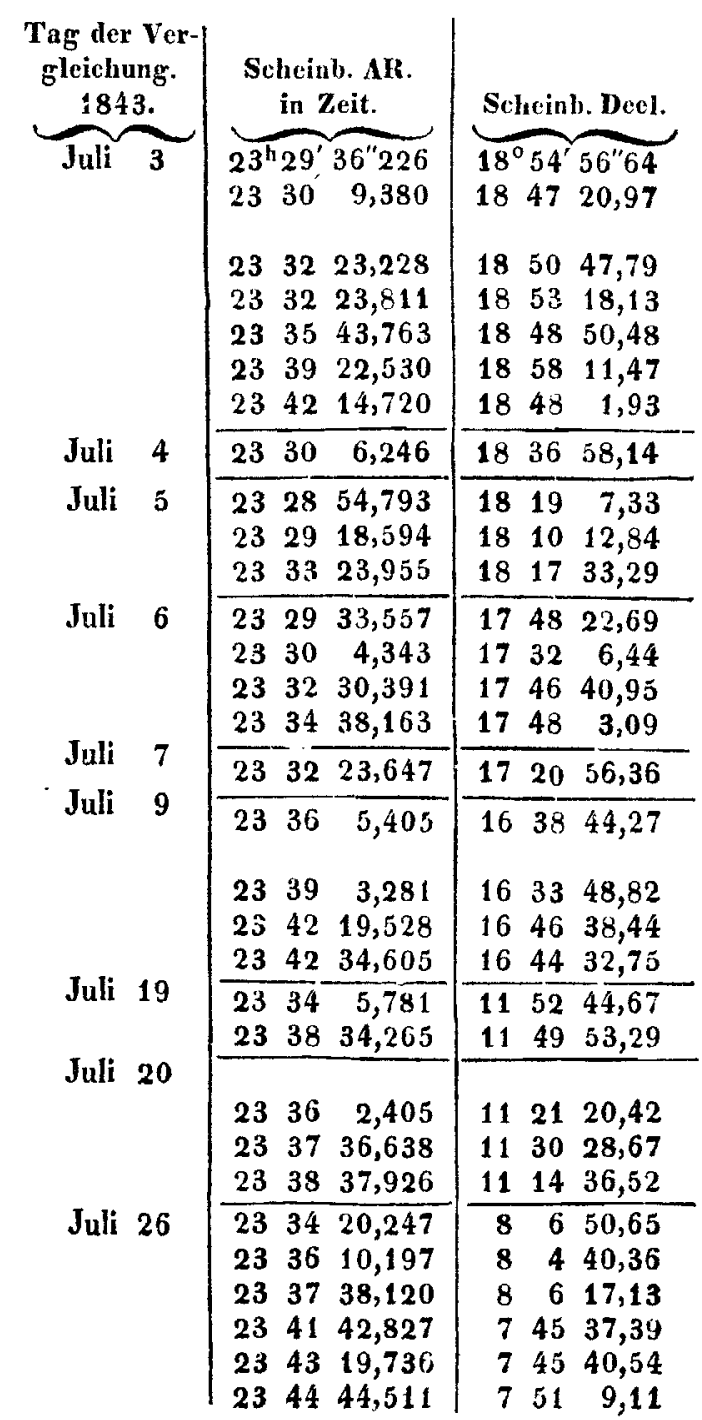

